\title{
Estudo comparativo da dispersão de nanotubos de carbono e sua adição em um cimento endodôntico à base de óxido de zinco e eugenol
}

\section{Comparative study of dispersion of carbon nanotubes and addition in zinc oxide-eugenol-based root canal sealer}

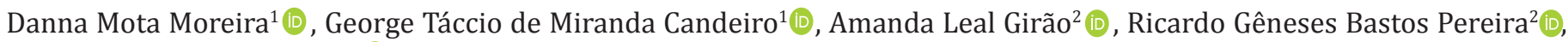 \\ Ana Trícia Soeiro Florêncio² ${ }^{2}$ \\ 1. Docente do Curso de Odontologia do Centro Universitário Christus (Unichristus), Fortaleza, CE, Brasil. 2. Discente do Curso de Odontologia do Centro \\ Universitário Christus (Unichristus), Fortaleza, CE, Brasil
}

\section{Resumo}

\begin{abstract}
Objetivo: avaliar a durabilidade da dispersão de nanotubos de carbono (NTC) em dodecil sulfato de sódio (SDS) com o intuito de analisar possíveis melhorias no módulo de elasticidade de um cimento endodôntico à base de óxido de zinco e eugenol. Métodos: após a dispersão dos NTC em SDS $2 \%, 25 \mu \mathrm{L}$ da solução de NTC+SDS foi adicionada ao cimento Endométhasone N (EMTN). 10 corpos-de-prova foram confeccionados de acordo com os seguintes grupos e tempos após a dispersão (n=10): G1 (Controle): EMTN; G2: EMTN + (NTC+SDS) imediato; G3: EMTN + (NTC+SDS) 24 horas; G4: EMTN + (NTC+SDS) 7 dias e G5: EMTN + (NTC+SDS) 1 mês. As amostras foram submetidas ao teste de flexão de 3-pontos para análise do módulo de elasticidade e os dados foram submetidos à análise estatística. Resultados: não houve diferença significante entre o grupo controle $(957,8 \pm 170 \mathrm{MPa})$ e o grupo imediato $(952,6$ $\pm 93,5 \mathrm{MPa})(\mathrm{p}>0,05)$. Já os grupos 24 horas $(700,2 \pm 84,9 \mathrm{MPa})$ e 7 dias $(727,5 \pm 93 \mathrm{MPa})$ demonstraram diferença significante em relação ao grupo controle $(p<0,05)$. 0 grupo 1 mês $(545 \pm 165 \mathrm{MPa})$ mostrou diferença significante em relação ao grupo controle e ao grupo Imediato ( $<<0,05)$. Conclusão: a incorporação de NTC ao cimento Endométhasone N não proporcionou melhorias no módulo de elasticidade quando da sua dispersão imediata ao uso. Entretanto, houve declínio do módulo de elasticidade quando do uso da solução de NTC dispersos em SDS $2 \%$ após o período de 24 horas, 7 dias e 1 mês.
\end{abstract}

Palavras-chave: Nanotubos de carbono. Endodontia. Módulo de elasticidade.

\begin{abstract}
Objective: to evaluate the stability of carbon nanotubes (NTC) suspensions in sodium dodecyl sulfate (SDS) in order to analyze possible improvements in the elastic modulus of a zinc oxide-eugenol-based root canal sealer. Methods: after dispersion of NTC in 2\% SDS, $25 \mu \mathrm{L}$ of the NTC + SDS solution was added to the Endométhasone N (EMTN). 10 specimens were prepared according to the following groups and times after dispersion ( $\mathrm{n}=10)$ : G1 (Control): EMTN; G2: EMTN + (NTC + SDS) immediate; G3: EMTN + (NTC + SDS) 24 hours; G4: EMTN + (NTC + SDS) 7 days and G5: EMTN + (NTC + SDS) 1 month. The samples were submitted to the 3-point bending test to analyze the elastic modulus and the data were submitted to statistical analysis. Results: there was no significant difference between the control group (957.8 $\pm 170 \mathrm{MPa})$ and G2 (952.6 $\pm 93.5 \mathrm{MPa})(\mathrm{p}>0.05)$. The G3 (700.2 $\pm 84.9 \mathrm{MPa})$ and G4 (727.5 $\pm 93 \mathrm{MPa}$ ) showed a significant difference in relation to the control group ( $\mathrm{p}<0.05)$. The G5 $(545 \pm 165 \mathrm{MPa})$ showed a significant difference in relation to the control group and the G2 (p < 0.05). Conclusion: the incorporation of the immediate dispersion of NTC to Endométhasone N did not change or provide improvements in the elastic modulus. However, there was a decrease in the elastic modulus after the period of 24 hours, 7 days and 1 month.
\end{abstract}

Key words: Carbon nanotubes. Endodontics. Elastic modulus.

INTRODUÇÃO

A busca por melhorias em todas as etapas operatórias da terapia endodôntica têm estimulado o desenvolvimento de estudos com o objetivo de aprimorar desde o diagnóstico, passando por técnicas e materiais obturadores que venham a trazer melhores índices de sucesso. Como parte desta busca por inovação e sucesso no tratamento endodôntico, melhorias nos materiais obturadores vêm sendo pesquisadas e lançadas no mercado ${ }^{1,2}$.

Os cimentos endodônticos são de fundamental importância para o selamento do canal radicular pois ficam em contato direto com o tecido conjuntivo apical e periapical, podendo causar reações teciduais. Vários fatores podem interferir na reação tecidual, favorável ou desfavoravelmente, entre os quais a composição química do cimento obturador ${ }^{3}$. Assim, o desenvolvimento de estudos à procura de um cimento obturador ideal, com menor potencial irritativo, indutor de um selamento apical, com boas propriedades físico-químicas e biocompatível é considerado imprescindível. O estudo das propriedades viscoelásticas de um cimento endodôntico traz importantes informações sobre seu comportamento dentro do ambiente do sistema de canais 
radiculares e possíveis melhorias podem melhorar os índices de sucesso da terapia endodôntica ${ }^{1,3}$.

Dentre as novas tecnologias que permitem melhorias aos materiais odontológicos, podemos citar o advento dos nanomateriais. Os materiais nanoestruturados tem despertado interesse da comunidade científica devido às suas impressionantes propriedades físicas e mecânicas. Os materiais e dispositivos produzidos em nanoescala $(10-9 \mathrm{~m})$ apresentam propriedades físico-químicas diferenciadas devido ao aparecimento de efeitos quânticos, redução de tamanho e aumento da área interfacial, proporcionando diversas possibilidades a diferentes segmentos da ciência ${ }^{4,5}$.

Entre os materiais criados dentro do conceito de nanotecnologia estão uma classe de materiais nanoestruturados de carbono, tais como: fulerenos sintetizados em 1985 e os nanotubos de carbono descobertos por ljima em $1991^{6}$. Os nanotubos de carbono (NTC) possuem um grande potencial na Odontologia devido às suas propriedades únicas tais como baixo peso, alta condutividade elétrica, estabilidade química elevada, alta condutividade térmica e uma grande superfície de contato. Também apresentam extraordinárias propriedades mecânicas como resistência à tração de 50-100 GPa e módulo de elasticidade de 1-2 TPA ${ }^{5,7}$.

Os nanotubos de carbono são alótropos do carbono com uma nanoestrutura cilíndrica. Estes cilindros de moléculas de carbono possuem propriedades incomum e que são de altíssimo valor no campo da nanotecnologia, eletrônica, óptica e outros campos tecnológicos da ciência dos materiais. Os nanotubos de carbono têm sido considerados como potenciais candidatos para atuarem como agentes de reforço em polímeros possibilitando inúmeras melhorias nas estruturas dos materiais ${ }^{8}$.

Contudo, um grande desafio encontrado ao se utilizar tal nanomaterial é a sua adequada dispersão. Em diversas das suas importantes aplicações, os nanotubos de carbono somente apresentam suas propriedades únicas se estiverem em uma dispersão altamente homogênea. No entanto, são pouco solúveis na maioria dos solventes. Devido à forte atração de van der Waals, eles exibem uma tendência a se agregarem e formarem grandes agregados de difícil dispersão,10,11.

Uma ampla variedade de surfactantes (aniônico, catiônico e não iônico) têm sido estudada para dispersões de NTC, como lauril sulfato de sódio (SDS), dodecil benzeno sulfato de sódio (SDBS), brometo de cetiltrimetil amônio (CTAB), brometo de dodeciltrimetil amônio (DTAB) e a família dos surfactantes Tween. Os estudos indicam que os surfactantes produzem um eficiente recobrimento da parede dos NTC e induzem repulsões eletroestáticas e/ou estéricas, que poderiam contrabalancear as atrações de van der Waals entre os NTC e possibilitar o seu uso na área de biomateriais ${ }^{9,11}$.

De acordo com Silva et al. ${ }^{11}$, no intuito de obter dispersões homogêneas, duas alternativas diferentes são atualmente utilizadas para dispersar os nanotubos de carbono: os métodos mecânicos (físicos) e métodos químicos. Os métodos mecânicos usam a ultrassonicação e agitação, o que permite a separação dos nanotubos uns dos outros. Contudo, tais métodos fragmentam os nanotubos durante o processo, causando a diminuição da razão de aspecto, sendo considerados muitas vezes ineficientes, além de consumir tempo e energia. Já os métodos químicos usam surfactantes ou modificações químicas para alterar a energia de superfície dos NTC, melhorando as características de molhabilidade e adesividade, aumentando a estabilidade da dispersão no solvente. No entanto, funcionalizações químicas agressivas causam um aumento nos defeitos nas paredes laterais, o que pode alterar as propriedades mecânicas e elétricas dos NTC.

Assim, a escolha do dispersante ideal e o seu método de uso para os NTC continua uma incógnita. O dodecil sulfato de sódio (SDS), um composto orgânico aniônico, vem sendo testado como surfactante de escolha para a dispersão dos NTC visto, sua alta capacidade de promover dispersões estáveis dos NTC e por sua biocompatibilidade ${ }^{9,12}$.

Tendo em vista as promissoras possibilidades do uso de NTC, o presente estudo buscou realizar o aprimoramento de um cimento endodôntico do tipo óxido de zinco e eugenol, o Endométhasone N (Septodont Brasil LTDA, SP, Brasil) por meio da adição de nanotubos de carbono dispersos em SDS. $\mathrm{O}$ Endométhasone $\mathrm{N}$, apesar de não ser muito utilizado no Brasil, é bastante utilizado em outros países, principalmente pela presença de corticosteróide na formulação. No presente estudo, a sua escolha também se deu por permitir adição direta dos NTC sem a necessidade de processos complexos. Assim, a hipótese nula é de que a adição de nanotubos de carbono ao cimento Endométhasone $\mathrm{N}$ não irá reduzir o módulo de elasticidade de tal cimento endodôntico.

\section{MATERIAL E MÉTODOS}

Para a realização deste estudo os seguintes materiais foram utilizados:

- Cimento endodôntico à base de óxido de zinco e eugenol, Endométhasone N (Septodont Brasil LTDA, SP, Brasil).

- Os nanotubos de carbono (NTC) funcionalizados do tipo múltiplas paredes (Multi-Walled) doados pelo Laboratório de Crescimento Nanotubos de Carbono da Universidade Federal do Ceará. Os NTC utilizados possuíam diâmetros variando de 10 a $40 \mathrm{~nm}$ e comprimento majoritário entre 3 a $6 \mu \mathrm{m}$. Foram submetidos a um refluxo com ácido nítrico na concentração de $9 \mathrm{~mol} / \mathrm{L}$ por um período de 12 horas na temperatura de $175 \circ \mathrm{C}$ resultando em um material com pureza $>95 \%$ e área superficial de $320 \mathrm{~m}^{2} / \mathrm{g}$.

- Dodecil sulfato de sódio (SDS, 90\%, Vetc Química Fina LTDA, Rio de Janeiro, Brasil).

\section{Dispersão dos nanotubos de carbono em SDS}

A dispersão dos NTC em SDS foi feita de acordo com estudo de 
Bai et $a^{13}$. A proporção de 2,0g de NTC para $100 \mathrm{~mL}$ de SDS $2 \%$ foi utilizada. A solução foi mantida por 3 ciclos de 5 minutos no sonicador (Branson Digital Sonifier 450D, 400 W, 20 kHz) com amplitude de $10 \%$. Esta mesma solução foi adicionada ao cimento endodôntico e testada nos seguintes tempos: imediata, 24 horas, 7 dias e 1 mês. A solução foi mantida em estufa na temperatura de $3700 \mathrm{C}$ até o momento do seu uso prédeterminado.

\section{Ensaio de flexão em 3 pontos}

Para a realização do ensaio mecânico do módulo de elasticidade, um total de 10 corpos-de-prova de cimento endodôntico Endométhasone $\mathrm{N}$ foram confeccionados. $\mathrm{O}$ cimento recém manipulado foi inserido em uma matriz retangular $(25 \times 2 \times$ $2 \mathrm{~mm}$ ). Essa matriz foi mantida apoiada sobre uma placa de vidro e coberta com uma tira de poliéster. As amostras foram divididas em 5 grupos $(n=10)$, conforme descrito abaixo:

Grupo 1 - Controle - Endométhasone $\mathrm{N}$ manipulado de acordo com as instruções do fabricante.

Grupo 2 - Endométhasone N + nanotubos de carbono/SDS (imediata).

Grupo 3 - Endométhasone N + nanotubos de carbono/SDS (24 horas).

Grupo 4 - Endométhasone N + nanotubos de carbono/SDS (7 dias).

Grupo 5 - Endométhasone N + nanotubos de carbono/SDS (1 mês).

Para a confecção das amostras, a seguinte proporção foi utilizada: $0,33 \mathrm{~g}$ do pó do Endométhasone para 4 gotas de eugenol e $25 \mu \mathrm{L}$ da solução de NTC + SDS $2 \%$. Os componentes foram espatulados em placa de vidro até a sua completa homogeneização. As amostras ficaram mantidas nos moldes em placas de vidro e armazenadas em estufa $37^{\circ} \mathrm{C}$ em $100 \%$ de umidade por um período mínimo de 48 horas a fim de que a presa do cimento endodôntico fosse obtida.

O ensaio mecânico para registro e cálculo do módulo de elasticidade foi realizado em uma Máquina de Ensaios Universal (Instron 4411, Norwood, MA, EUA) por meio de um teste de carga de três pontos. Para a realização do ensaio, foi utilizado um dispositivo metálico específico para este teste, contendo duas barras fixas dispostas paralelamente entre si e distantes $18 \mathrm{~mm}$, onde as amostras foram apoiadas. $O$ teste foi executado com o auxílio de uma célula de carga de $500 \mathrm{~N}$ com velocidade constante em $0,5 \mathrm{~mm} / \mathrm{min}$.

Os espécimes foram submetidos à carga compressiva até a fratura e os resultados analisados em software específico (Bluehill 2 software, Instron Corporation, Norwood, MA, EUA) que informaram os valores do módulo de elasticidade em MPa.

\section{Análise estatística}

Os dados foram tabulados no Microsoft Excel e exportados para o software Statistical Packcage for the Social Sciences (SPSS) versão 17,0 para Windows. Os valores obtidos foram submetidos à análise de variância (ANOVA). Diferenças entre os grupos foram avaliadas pelo método de comparação múltipla de Bonferroni. Os dados foram expressos em forma de média e desvio-padrão e consideradas estatisticamente significantes a partir de $p<0,05$.

\section{RESULTADOS}

No presente estudo o dodecil sulfato de sódio (SDS) foi o surfactante de escolha para a dispersão dos NTC. Foi possível observar uma dispersão homogênea sem maiores modificações visuais por até 1 mês de observação (Figura 1).

Figura 1. Solução de NTC + SDS $2 \%$ após 1 mês da sua dispersão.

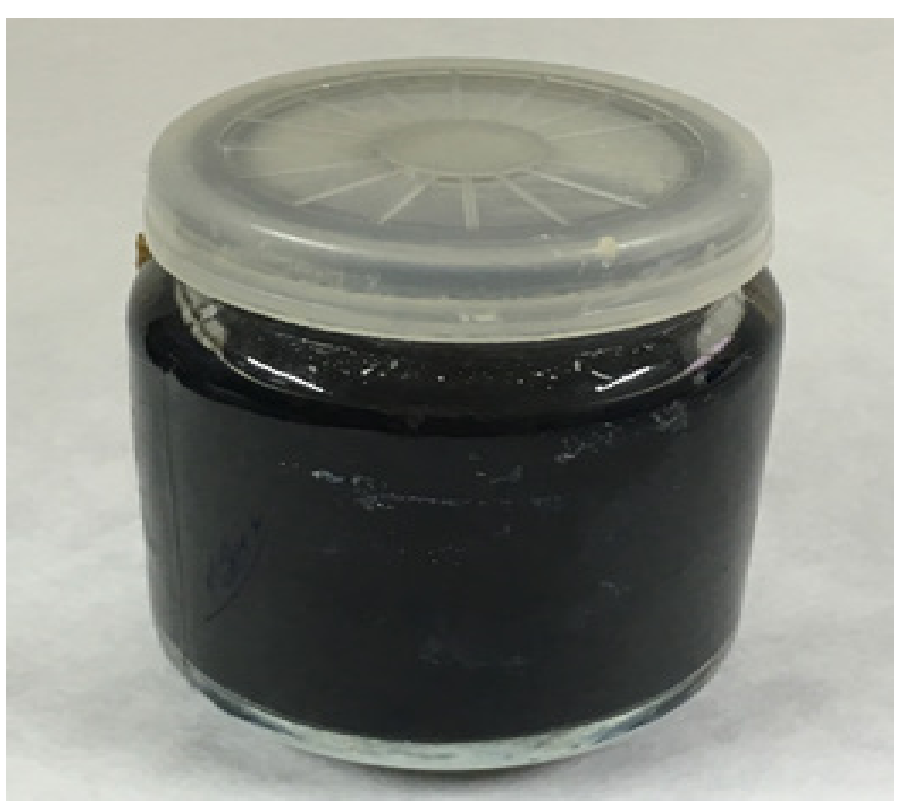

Em relação ao ensaio mecânico de 3 pontos para análise do módulo de elasticidade (Tabela 1), os resultados mostraram que não houve diferença significante entre o grupo controle $(957,8$ $\pm 170 \mathrm{MPa})$ e o grupo imediato $(952,6 \pm 93,5 \mathrm{MPa})(\mathrm{p}>0,05)$. O módulo de elasticidade dos grupos 24 horas $(700,2 \pm 84,9$ $\mathrm{MPa}$ ) e 7 dias $(727,5 \pm 93 \mathrm{MPa})$ mostrou diferença significante em relação ao grupo controle $(p<0,05)$. Já o grupo 1 mês $(545$ $\pm 165 \mathrm{MPa}$ ) mostrou diferença significante em relação ao grupo controle e ao grupo Imediato $(p<0,05)$ (Figura 2$)$.

Tabela 1. Médias e desvio-padrão do Módulo de Elasticidade (Mpa) dos diferentes grupos.

\begin{tabular}{lllllll}
\hline \multirow{2}{*}{ Grupo } & \multirow{2}{*}{ Controle } & \multicolumn{5}{c}{ NTC } \\
\cline { 3 - 6 } & & Imediato & 24h & 7d & 1 mês & p-Valor \\
\hline Média & 957,8 & 952,6 & $700,2^{*}$ & $727,5^{*}$ & $545,0^{*+}$ & $<0,001$ \\
DP & 170,0 & 93,5 & 84,9 & 93,0 & 165,0 & \\
\hline *p<0,05, & versus & Controle; & $+\mathrm{p}<0,05$, & versus & imediato & (ANOVA/ \\
\multicolumn{3}{c}{ Bonferroni). Dados expressos em forma de média e desvio-padrão. }
\end{tabular}


Figura 2. Médias e desvio-padrão do Módulo de Elasticidade (MPa) diferentes grupos. ${ }^{*} p<0,05$, versus Controle; ${ }^{t} p<0,05$, versus imediato (ANOVA/Bonferroni). Dados expressos em forma de média e desvio-padrão.

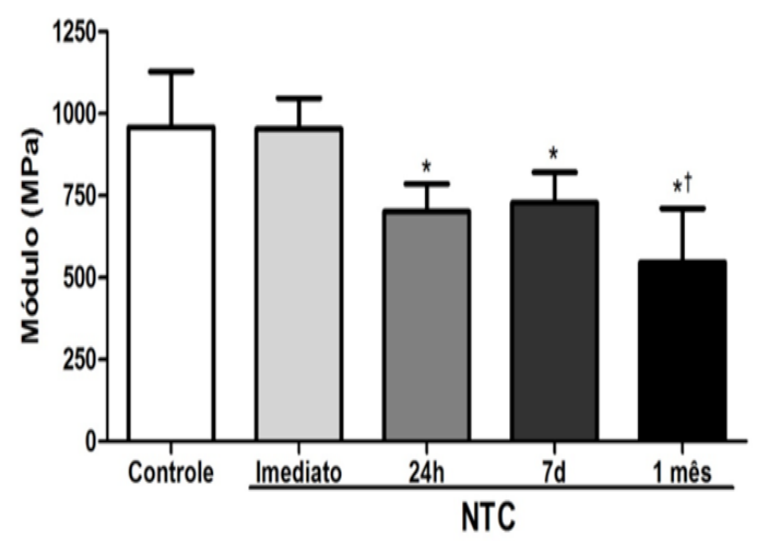

\section{DISCUSSÃO}

O presente estudo teve como objetivo procurar o aprimoramento de um cimento endodôntico do tipo óxido de zinco e eugenol de ampla utilização mundial por meio da adição de nanotubos de carbono buscando assim, obter melhorias nas propriedades mecânicas e biológicas de tal cimento. O presente trabalho é um dos pioneiros de uma linha de pesquisa do uso de nanotubos de carbono adicionados a materiais endodônticos na busca de uma melhor performance dos mesmos. A importância de tal estudo advém de um melhor conhecimento das possíveis utilizações de tais nanomateriais, as dificuldades enfrentadas e as soluções para o seu real uso, o que abre uma série de possibilidades para o aprimoramento de outros tipos de cimentos endodônticos e desenvolvimento de novos materiais.

O Endométhasone $\mathrm{N}$, apesar de não ser muito utilizado no Brasil, é bastante utilizado em outros países, principalmente pela presença de corticosteróide na formulação e por permitir adição direta dos NTC sem necessidade de modificação da proporção de seus componentes originais ou mesmo processos complexos de adição. O dodecil sulfato de sódio (SDS), um composto orgânico aniônico, foi o surfactante de escolha para a dispersão dos NTC visto, sua alta capacidade de promover dispersões estáveis dos NTC e por sua biocompatibilidade ${ }^{9,12}$. A natureza do surfactante, sua concentração e tipo de interação são conhecidos por desempenhar um papel crucial no comportamento de fase dos nanotubos de carbono ${ }^{14}$.

O uso do SDS como surfactante se torna interessante visto que a região hidrofóbica dessas moléculas interage com a superfície dos nanotubos, destruindo a interface hidrofóbica dos nanotubos com a água e a interação tubo-tubo (van der Waals) responsável pela agregação em feixes. As micelas formadas por surfactante e nanotubos formam dispersões estáveis em meio aquoso ${ }^{15}$.

Estudo de Bai et al. $^{13}$ mostrou a possiblidade de ação antimicrobiana quando da dispersão de NTC multi-paredes (Multi-Walled) em SDS. O que torna ainda mais interessante e promissor a escolha por tal surfactante, principalmente em materiais odontológicos, apesar de no presente estudo nenhum teste de ação antimicrobiana tenha sido realizado.

Os resultados do presente estudo mostraram que houve diferença significante entre o grupo controle e os grupos 24 horas, 7 dias e 1 mês, além deste último também ter demonstrado diferença significante em relação ao grupo imediato. Tais resultados indicam, inicialmente, a necessidade de dispersão imediata dos nanotubos de carbono quando da sua utilização para que não haja decréscimo da propriedade mecânica testada. Assim, a hipótese nula se confirma apenas para o grupo da dispersão imediata dos NTC.

Os efeitos negativos dos nanotubos de carbono adicionados ao Endométhasone $\mathrm{N}$ nos períodos avaliados de 24 horas, 7 dias e 1 mês de dispersão, possivelmente, tenham ocorrido por falhas neste processo. Um primeiro aspecto a ser analisado, ao considerar a redução da propriedade mecânica testada, seria a concentração de $2 \%$ de SDS utilizada no presente estudo. Tal concentração diferiu do estudo de Bai et al. ${ }^{13}$, o qual usou $1 \%$ de SDS. Possivelmente, a concentração de $2 \%$ não permita a estabilidade e expressão adequado das propriedades dos NTC diante dos diferentes períodos testados.

A própria proporção de NTC utilizada na dispersão em SDS, possivelmente, seja outro fator problemático para que suas excelentes propriedades tenham sua expressão adequada. Estudo realizado por Santos et al. ${ }^{16}$, fez a adição nanotubos de carbono em diferentes cimentos de ionômero de vidro e também não tiveram êxito neste intento, provavelmente, por falhas na concentração de NTC utilizada.

Resultado diferente foi obtido em pesquisa que fez a adição de NTC em resina acrílica à base de polimetilmetacrilato quando da avaliação da contração de polimerização. Os autores relataram redução da contração de polimerização quando realizada adições de NTC em diferentes proporções, sendo a proporção de $0,5 \%$ a que obteve o melhor resultado ${ }^{8}$.

No presente estudo foi observado um decréscimo nos valores do módulo de elasticidade após 24 horas, uma semana, e um mês de manipulação/dispersão da solução de NTC + SDS. Turagam \& Mudrakola ${ }^{8}$, em estudo prévio utilizando nanotubos de carbono, demonstrou que o uso imediato da dispersão de NTC em monômero de polimetilmetacrilato foi, à princípio, a melhor opção como mostrado também no presente estudo.

Outros trabalhos publicados utilizaram a adição imediatamente a manipulação, o que foi confirmado como sendo, à princípio, a melhor opção como mostrado no presente estudo.

Uma observação interessante do presente estudo consiste que não houve alteração de cor do cimento Endométhasone $\mathrm{N}$ em nenhum dos grupos experimentais após a adição da 
solução de NTC + SDS, que possui uma coloração escura (preta). Provavelmente, tal fato tenha se dado pela pequena quantidade de solução adicionada em cada amostra.

Ainda existem muitas dificuldades relacionadas a utilização do nanotubos de carbono, devido ao pouco conhecimento da proporção ideal para que as propriedades positivas do nanotubos sejam expressadas. Também se desconhece a melhor forma de dispersão e o tipo de surfactante que melhor se adequaria a cada situação de uso dos NTC, tornando isso uma limitação de se trabalhar com esse material, sendo necessário primeiramente obter esses conhecimentos para que seja possível trabalhar de forma positiva com os nanotubos de carbono adicionados aos materiais odontológicos.

Dentro da limitação do presente estudo, recomenda-se que a dispersão dos nanotubos deve ser feita imediatamente a utilização destes. Desta forma, novos estudos devem ser conduzidos a fim de determinar o surfactante ideal, bem como a proporção correta para que melhorias nas propriedades mecânicas e biológicas de cimentos endodônticos à base de óxido de zinco e eugenol possam ser implementadas. Testes adicionais também são necessários relativos a possíveis modificações nos tempos de trabalho e presa e modificações em outras propriedades mecânicas.

\section{CONCLUSÃO}

A incorporação de NTC ao cimento Endométhasone $\mathrm{N}$ não proporcionou melhorias no módulo de elasticidade quando da sua dispersão imediata ao uso. Entretanto, houve declínio do módulo de elasticidade quando do uso da solução de nanotubos de carbono dispersos em SDS $2 \%$ após o período de 24 horas, 7 dias e 1 mês, afetando negativamente o cimento Endométhasone $\mathrm{N}$.

\section{AGRADECIMENTOS}

Os autores agradecem ao Programa de Pós-Graduação em Odontologia da Universidade Federal do Ceará pelo apoio técnico a esta pesquisa.

\section{REFERÊNCIAS}

1. Holland R, Gomes JE Filho, Cintra LTA, Queiroz ÍOA, Estrela C. Factors affecting the periapical healing process of endodontically treated teeth. J Appl Oral Sci. 2017 Sep-Oct; 25(5):465-476. PubMed PMID: 29069143.

2. Silva Almeida LH, Moraes RR, Morgental RD, Pappen FG. Are Premixed Calcium Silicate-based Endodontic Sealers Comparable to Conventional Materials? A Systematic Review of In Vitro Studies. J Endod. 2017 Apr;43(4):527-535. PubMed PMID: 28216270.

3. Kaplan AE, Ormaechea MF, Picca M, Canzobre MC, Ubios AM. Rhealogical properties and biocompatibility of endodontic sealers. Int Endod J. 2003 Aug; 36(8):527-32. PubMed PMID: 12887381.

4. Lemay SG, Janssen JW, van den Hout M, Mooij M, Bronikowski MJ, Willis PA, Smalley RE, Kouwenhoven LP, Dekker C. Two-dimensional imaging of electronic wavefunctions in carbon nanotubes. Nature. 2001 Aug; 412(6847):617-20. PubMed PMID: 11493914.

5. Tonelli FM, Santos AK, Gomes KN, Lorençon E, Guatimosim S, Ladeira LO, Resende RR. Carbon nanotube interaction with extracellular matrix proteins producing scaffolds for tissue engineering. Int J Nanomedicine. 2012 Aug;7:4511-29. PubMed PMID: 22923989.

6. lijima S. Helical Microtubules of Graphitic Carbon. Nature. 1991; 354(6348): 56-58.

7. Zhang $F, X i a ~ Y, X u L, G u N$. Surface modification and microstructure of singlewalled carbon nanotubes for dental resin-based composites. J Biomed Mater Res B Appl Biomater. 2008 Jul; 86(1):90-7. PMID: 18098184.

8. Turagam N, Mudrakola DP. Effect of micro-additions of carbon nanotubes to polymethylmethacrylate on reduction in polymerization shrinkage. J Prosthodont. 2013 Feb; 22(2):105-11. PubMed PMID: 22985308.
9. Yu J, Grossiord N, Koning CE, Loos J. Controlling the dispersion of multiwall carbon nanotubes in aqueous surfactant solution. Carbon. $2007 \mathrm{Mar}$; 45(3):618-23.

10. Ma PC, Siddiqui NA, Marom G, Kim JK. Dispersion and functionalization of carbon nanotubes for polymer-based nanocomposites: A review. Composites: Part A. 2010 Oct; 41(10):1345-1367.

11. Silva IR, Barreto PLM, Bellettini IC. Estudo das dispersões aquosas de nanotubos de carbono utilizando diferentes surfactantes. Quim. Nova. 2013 Nov; 36(1): 5-9.

12. Bystrzejewski M, Huczko A, Lange $H$, Gemming T, Büchner B, Rümmeli MH. Dispersion and diameter separation of multi-wall carbon nanotubes in aqueous solutions. J Colloid Interface Sci. 2010 May; 345(2):138-42. PubMed PMID: 20171648.

13. Bai Y, Park IS, Lee SJ, Bae TS, Watari F, Uo M, Lee MH. Aqueous dispersion of surfactant-modified multiwalled carbon nanotubes and their application as an antibacterial agent. Carbon. 2011 Sept; 49:3663-3671.

14. Vaisman L, Wagner HD, Marom G. The role of surfactants in dispersion of carbon nanotubes. Adv Colloid Interface Sci. 2006 Dec 21;128-130:37-46. PubMed PMID: 17222381.

15. Souza-Filho AG, Fagan SB. Funcionalização de nanotubos de carbono. Quim. Nova. 2007 Sept; 30(7): 1695-1703.

16. Santos MMPR, Mathias IF, Diniz MB, Bresciani E. Avaliação da dureza superficial de cimentos de ionômero de vidro reforçados por nanotubos de carbon. Rev Odontol UNESP. 2015 Mar-Apr; 44(2): 108-112. 